ARTICLE

Received 18 May 2014 | Accepted 3 Oct 2014 | Published 13 Nov $2014 \quad$ DOl: 10.1038/ncomms6478

\title{
Thickness sorting of two-dimensional transition metal dichalcogenides via copolymer-assisted density gradient ultracentrifugation
}

Joohoon Kang¹, Jung-Woo T. Seo ${ }^{1}$, Diego Alducin², Arturo Ponce ${ }^{2}$, Miguel Jose Yacaman² \& Mark C. Hersam¹,3,4

Two-dimensional transition metal dichalcogenides have emerged as leading successors to graphene due to their diverse properties, which depend sensitively on sample thickness. Although solution-based exfoliation methods hold promise for scalable production of these materials, existing techniques introduce irreversible structural defects and/or lack sufficient control over the sample thickness. In contrast, previous work on carbon nanotubes and graphene has shown that isopycnic density gradient ultracentrifugation can produce structurally and electronically monodisperse nanomaterial populations. However, this approach cannot be directly applied to transition metal dichalcogenides due to their high intrinsic buoyant densities when encapsulated with ionic small molecule surfactants. Here, we overcome this limitation and thus demonstrate thickness sorting of pristine molybdenum disulfide $\left(\mathrm{MoS}_{2}\right)$ by employing a block copolymer dispersant composed of a central hydrophobic unit flanked by hydrophilic chains that effectively reduces the overall buoyant density in aqueous solution. The resulting solution-processed monolayer $\mathrm{MoS}_{2}$ samples exhibit strong photoluminescence without further chemical treatment.

\footnotetext{
${ }^{1}$ Department of Materials Science and Engineering, Northwestern University, Evanston, Illinois 60208, USA. ${ }^{2}$ Department of Physics and Astronomy, University of Texas at San Antonio, San Antonio, Texas 78249, USA. ${ }^{3}$ Department of Chemistry, Northwestern University, Evanston, Illinois 60208 , USA.

${ }^{4}$ Department of Medicine, Northwestern University, Evanston, Illinois 60208, USA. Correspondence and requests for materials should be addressed to

M.C.H. (email: m-hersam@northwestern.edu).
} 
$\mathrm{M}$ olybdenum disulfide $\left(\mathrm{MoS}_{2}\right)$, one of the transition metal dichalcogenides (TMDs), has been studied with great interest for a wide range of applications due to its unique electronic, optical and catalytic properties ${ }^{1-15}$. The bulk $\mathrm{MoS}_{2}$ crystal is a semiconductor with a $1.2 \mathrm{eV}$ indirect bandgap and consists of covalently bonded S-Mo-S stoichiometric layers that interact with neighbouring layers via the relatively weak van der Waals interaction. When isolated as a single stoichiometric layer, $\mathrm{MoS}_{2}$ shows strong visible photoluminescence due to the emergence of a $1.9 \mathrm{eV}$ direct bandgap at the $\mathrm{K}$ point of the hexagonal Brillouin zone $e^{2,8,16}$. To fully understand and exploit the unique properties of single-layered $\mathrm{MoS}_{2}$, many production methods have been developed including micromechanical ${ }^{1}$, chemical $^{11,17}$ and surfactant-assisted liquid-phase exfoliation ${ }^{18-21}$, in addition to growth via chemical vapour deposition ${ }^{22}$. While the micromechanical cleavage technique produces single- or fewlayered $\mathrm{MoS}_{2}$ with high structural quality, this method lacks sufficient scalability for practical applications. On the other hand, solution-phase exfoliation of single-layered $\mathrm{MoS}_{2}$ can be achieved by lithium intercalation, although this process drives a phase transition to the metallic $1 \mathrm{~T}-\mathrm{MoS}_{2}$ structure, thus necessitating subsequent thermal treatments in an attempt to recover the semiconducting $2 \mathrm{H}-\mathrm{MoS}_{2}$ phase $\mathrm{s}^{11,23}$. However, complete semiconducting phase recovery has not yet been demonstrated, and the presence of the residual metallic phase can have detrimental effects, especially in electronic applications. Alternative liquid exfoliation methods can avoid the metallic 1T- $-\mathrm{MoS}_{2}$ phase transition, but typically yield a range of $\mathrm{MoS}_{2}$ flake thicknesses. Finally, ultrathin $\mathrm{MoS}_{2}$ growth by chemical vapour deposition requires high temperature processing and subsequent transfer from the growth substrate, which is arguably more cumbersome than solution-based approaches.

Here, we demonstrate an alternative solution-based route for preparing structurally pristine, ultrathin $\mathrm{MoS}_{2}$ nanosheets via isopycnic density gradient ultracentrifugation (DGU). Originally used for biomolecule separations ${ }^{24}$, DGU has emerged as one of the leading techniques for isolating structurally and electronically monodisperse populations of carbon nanotubes ${ }^{25-27}$ and graphene ${ }^{28}$. The physical structure of carbon nanomaterials coupled with their surrounding hydrated amphiphilic surfactants in aqueous solution leads to subtle buoyant density differences that drive separation to their respective isopycnic points when subjected to ultracentrifugation in a density gradient medium. In previous DGU studies on carbon nanomaterials, small molecule ionic surfactants, such as sodium cholate (SC), have proven to be exceptional dispersants that also enable exquisite control over the buoyant density as a function of the carbon nanomaterial physical and electronic structure ${ }^{28,29}$.

\section{Results}

Buoyant density model. To assess the applicability of DGU to $\mathrm{MoS}_{2}$ nanosheets, the following geometrical buoyant density model was used for SC-encapsulated $\mathrm{MoS}_{2}$ :

$$
\rho(N)=\frac{\rho_{\mathrm{S}} N+2 m_{\text {surf }} \sigma+2 \rho_{\mathrm{H}_{2} \mathrm{O}} t_{\mathrm{H}}}{(N+1) t_{\mathrm{Mos}_{2}}+2 t_{\mathrm{A}}+2 t_{\mathrm{H}}}
$$

where $\rho_{\mathrm{s}}=3.39 \times 10^{-7} \mathrm{~g} \mathrm{~cm}^{-2}$ is the sheet density of $\mathrm{MoS}_{2}, \mathrm{~N}$ is the number of the $\mathrm{MoS}_{2}$ sheets, $m_{\text {surf }}=7.15 \times 10^{-22} \mathrm{~g}$ is the mass of one SC molecule, $\sigma=1.35 \mathrm{~nm}^{-2}$ is the surface packing density of SC on $\mathrm{MoS}_{2}, \rho_{\mathrm{H} 2 \mathrm{O}}$ is the density of water, $t_{\mathrm{H}}=3.3 \mathrm{~nm}$ is the assumed hydration shell thickness, $t_{\mathrm{Mos} 2}=0.67 \mathrm{~nm}$ is the $\mathrm{MoS}_{2}$ interlayer distance, and $t_{\mathrm{A}}=0.355 \mathrm{~nm}$ is the assumed anhydrous shell thickness. The values for $\sigma, t_{\mathrm{H}}$, and $t_{\mathrm{A}}$ are based on previously established values for SC-encapsulated graphene ${ }^{28}$. The resulting buoyant densities are presented in Fig. 1c, where
SC-encapsulated $\mathrm{MoS}_{2}$, even in the monolayer limit, is predicted to be more dense than iodixanol, the density gradient medium, at its solubility limit $\left(1.32 \mathrm{~g} \mathrm{~cm}^{-3}\right)$. Evidently, the relatively large sheet density of $\mathrm{MoS}_{2}$ compared with graphene implies that the hydration of SC is insufficient to lower the buoyant density of suspended $\mathrm{MoS}_{2}$ nanosheets to a level compatible with isopycnic DGU.

To overcome this issue, we sought an alternative dispersant for $\mathrm{MoS}_{2}$ that would enable greater hydration and thus lower buoyant density in aqueous solution. The nonionic, biocompatible, amphiphilic block copolymer Pluronic F68 was selected due to its relatively large molecular weight, long hydrophilic chains for effective steric hindrance, and its compatibility with the DGU process $^{30}$. In particular, F68 is composed of a central hydrophobic polypropylene oxide (PPO) unit surrounded by hydrophilic polyethylene oxide chains (Fig. 1a), and is known to be effective as an amphiphilic dispersant for graphene ${ }^{31}$ and DGU separation of carbon nanotubes ${ }^{30}$. To estimate the buoyant density of F68-encapsulated $\mathrm{MoS}_{2}$, the parameters in the aforementioned model are modified as following: $m_{\text {surf }}=1.40 \times 10^{-20} \mathrm{~g}$ is the mass of one F68 molecule, $t_{\mathrm{A}}=1.6 \mathrm{~nm}, t_{\mathrm{H}}=20.6 \mathrm{~nm}$ and $\sigma$ is varied between 0.058 and $0.575 \mathrm{~nm}^{-2}$ to account for the two likely extremes where the surface coverage of PPO chains on the $\mathrm{MoS}_{2}$ surface ranges from $10-100 \%$. While this model is oversimplified, it suggests that the buoyant density of F68- $\mathrm{MoS}_{2}$ is substantially reduced compared with SC- $\mathrm{MoS}_{2}$ and likely to fall within the achievable range of iodixanol-based density gradients, especially in the limit of ultrathin $\mathrm{MoS}_{2}$ (Fig. 1c).

Thickness sorting via density gradient ultracentrifugation. To assess the effectiveness of F68-MoS ${ }_{2}$ for DGU, $1 \mathrm{~g}$ of $\mathrm{MoS}_{2}$ powder was first exfoliated and dispersed in $2 \%$ weight per volume $\left(\mathrm{wv}^{-1}\right)$ aqueous solution of F68 via tip ultrasonication (See Supplementary Discussion for details). After ultrasonication, we found that $17.1 \%$ of the initial $\mathrm{MoS}_{2}$ mass was dispersed, as determined by measuring the mass of the undispersed residual $\mathrm{MoS}_{2}$ slurry. The resulting F68-MoS 2 dispersion was placed in a step density gradient and ultracentrifuged at $32 \mathrm{kr}$.p.m. for $24 \mathrm{~h}$ to remove thick $\mathrm{MoS}_{2}$ flakes and enhance the population of thin $\mathrm{MoS}_{2}$ nanosheets (Fig. 2a). After this concentration step, $0.027 \mathrm{mg}$ of $\mathrm{MoS}_{2}$ flakes with thickness $<5 \mathrm{~nm}$ remain, of which $24 \%$ are single-layer $\mathrm{MoS}_{2}$ flakes as determined by AFM. This level of exfoliation is comparable to previous literature ${ }^{32}$ and may be improved with the development of emerging techniques such as shear mixing ${ }^{33,34}$ or ball milling ${ }^{35}$. After ultracentrifugation, dense iodixanol solution containing $2 \% \mathrm{wv}^{-1} \mathrm{~F} 68$ was injected above the sedimented aggregates at the bottom of ultracentrifuge tube to separate and allow fractionation of the concentrated $\mathrm{MoS}_{2}$ nanosheets. The extracted $\mathrm{F} 68-\mathrm{MoS}_{2}$ dispersion was stable for several months. We also observed that when concentrated $\mathrm{MoS}_{2}$ nanosheets were prepared using SC as the surfactant, there was no isolation of a SC-MoS 2 band (Supplementary Fig. 2), which further illustrates the effectiveness of F68 as a dispersant for $\mathrm{MoS}_{2}$.

After the concentration step, the fractionated $\mathrm{F} 68-\mathrm{MoS}_{2}$ solution was placed at the bottom of a linear density gradient and ultracentrifuged at $28 \mathrm{kr} . \mathrm{p} . \mathrm{m}$. for $12 \mathrm{~h}$. The first iteration of DGU induces the F68- $\mathrm{MoS}_{2}$ nanosheets to separate into multiple visible bands throughout the ultracentrifuge tube (Fig. 2b). These bands were recovered in $1 \mathrm{~mm}$ steps using a piston gradient fractionator and labelled with their position (that is, fractions were recovered starting from $6 \mathrm{~mm}$ above the first band and thus f7 indicates the fraction that encompasses the first band). For further refinement, three fractions from the most well-defined bands (f7, f17 and f27) and relatively wider bands (f9, f13 and f23) were extracted, and then placed at the top of a linear density 

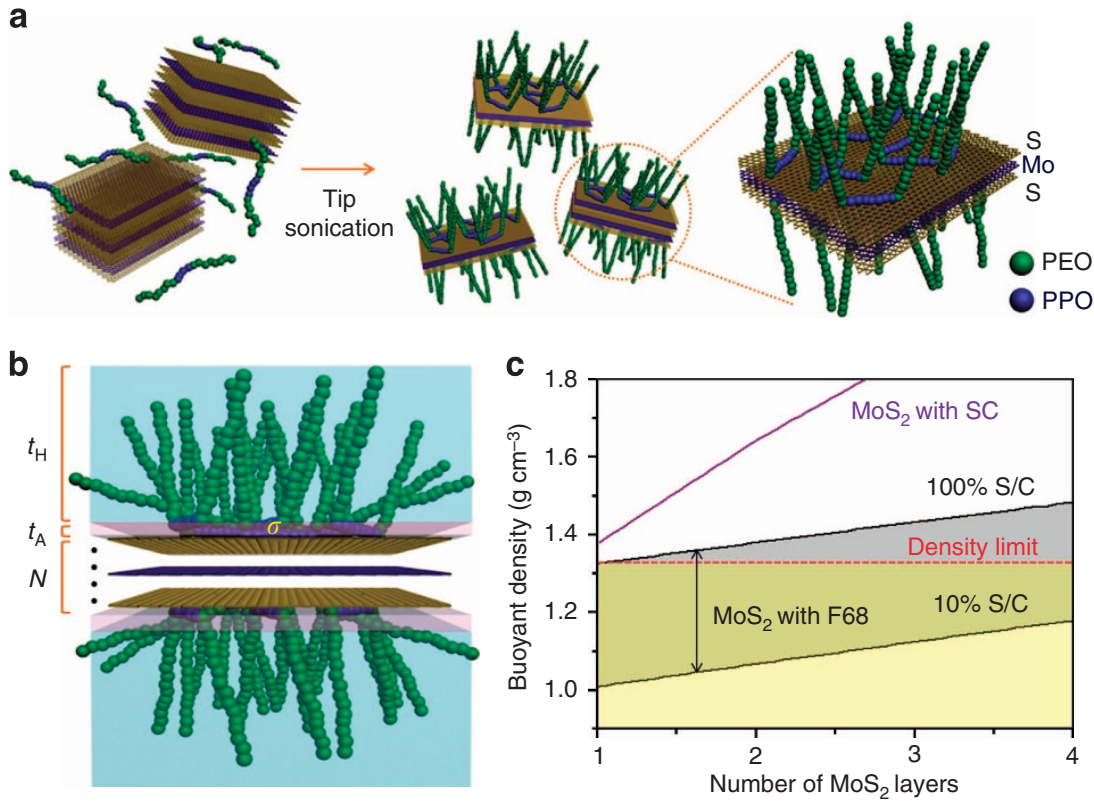

Figure 1 | Schematic illustration of the $\mathbf{M o S}_{\mathbf{2}}$ exfoliation process and buoyant density model. (a) Schematic of the MoS $\mathrm{S}_{2}$ exfoliation process in aqueous solution. In particular, $\mathrm{MoS}_{2}$ flakes are exfoliated and dispersed by Pluronic F68 during tip ultrasonication. The brown and purple atoms are sulfur and molybdenum, and the green and blue spheres are PEO and PPO chains, respectively. (b) Buoyant density model for F68-encapsulated MoS $\mathrm{S}_{2}$ in aqueous solution, where $N$ is the number of $\mathrm{MoS}_{2}$ layers, $t_{\mathrm{A}}$ is the anhydrous layer thickness, $t_{\mathrm{H}}$ is the hydration shell thickness, and $\sigma$ is the packing density.

(c) Buoyant density model as a function of $\mathrm{MoS}_{2}$ flake thickness. Black and purple lines represent scenarios where MoS $\mathrm{S}_{2}$ is encapsulated with $\mathrm{F} 68$ and sodium cholate (SC), respectively. The area enclosed by the two black lines indicates the possible buoyant density range that depends on the surface coverage of Pluronic F68 on $\mathrm{MoS}_{2}$. Isopycnic DGU separation of $\mathrm{MoS}_{2}$ is only possible when the surfactant-encapsulated MoS 2 nanosheets have a buoyant density below the density limit imposed by the density gradient medium (red dashed line).
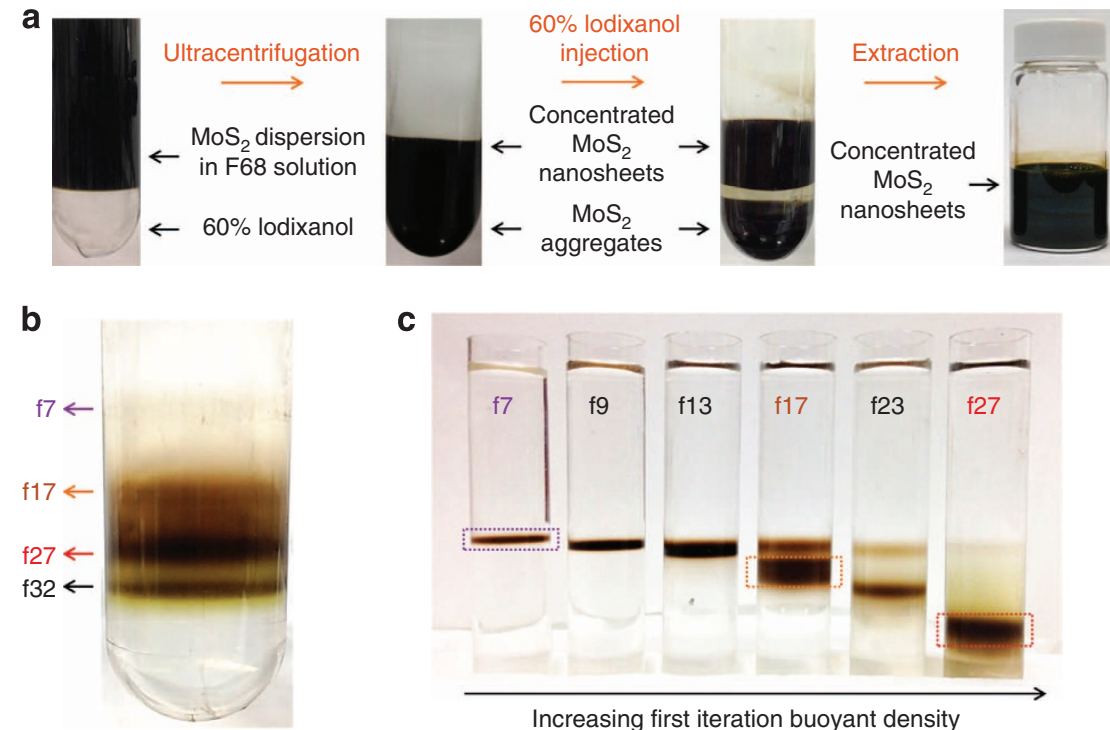

Figure 2 | Photographs of the experimental procedure. (a) Photographs of the concentration step for $\mathrm{MoS}_{2}$ nanosheets. After ultrasonication, the MoS 2 dispersion is placed on top of $60 \% \mathrm{w} \mathrm{v}^{-1}$ iodixanol as a step gradient and then ultracentrifuged at $32 \mathrm{kr}$.p.m. for $24 \mathrm{~h}$. Subsequently, $60 \% \mathrm{w} \mathrm{v}{ }^{-1}$ iodixanol is injected to separate the concentrated $\mathrm{MoS}_{2}$ nanosheets from the aggregates, and then extracted. The dispersion, gradient and injection solution all contain $2 \% \mathrm{wv}^{-1} \mathrm{~F} 68$. (b) Photograph of $\mathrm{MoS}_{2}$ bands in an ultracentrifuge tube after the first iteration of density gradient ultracentrifugation (DGU). The concentrated $\mathrm{MoS}_{2}$ solution was diluted by a factor of eight before ultracentrifugation to reveal the position of each band more clearly. (c) Photograph of the ultracentrifuge tubes after the second iteration of DGU. Fractions from the well-defined bands ( $f 7, f 17$ and $f 27$ ) and the relatively wider bands ( $f 9$, f13 and f23) from the first iteration are collected and used for the second iteration. The fractions from the dashed region are used for characterization.

gradient. The second iteration of DGU was performed at $41 \mathrm{kr} . \mathrm{p} . \mathrm{m}$. for $12 \mathrm{~h}$, leading to the formation of well-defined $\mathrm{MoS}_{2}$ bands at their corresponding isopycnic points (dashed regions in Fig. 2c), which were then fractionated for characterization. The topmost band, which will later be shown to be highly enriched in single-layer $\mathrm{MoS}_{2}$, possesses a mass of $0.002 \mathrm{mg}$, which implies that DGU captures $30.8 \%$ of the exfoliated singlelayer $\mathrm{MoS}_{2}$ flakes (See Supplementary Discussion for more details on the calculation of the DGU yield). It should be noted that similar DGU results were achieved for other TMDs (for example, 
DGU of $\mathrm{WS}_{2}, \mathrm{MoSe}_{2}$ and $\mathrm{WSe}_{2}$ are shown in Supplementary Figs 3 and 4), thus demonstrating the generality of the copolymer-assisted DGU for thickness sorting of two-dimensional nanomaterials.

Structure analysis of sorted MoS2 nanosheets. High-angle annular dark field scanning transmission electron microscopy (HAADF-STEM) was used to verify the $2 \mathrm{H}$ structural phase and characterize atomic-level defects of the $\mathrm{MoS}_{2}$ nanosheets. Figure $3 \mathrm{a}$ shows a schematic illustration of the $2 \mathrm{H}$ structural phase with trigonal prismatic coordination. A Fast Fourier Transform from the HAADF-STEM image with two direction index is shown in Fig. 3b. Figure $3 \mathrm{c}, \mathrm{d}$ show high resolution HAADF-STEM images of $\mathrm{MoS}_{2}$ nanosheets before and after DGU. The inset in Fig. 3d shows the magnified STEM image with the position of Mo (blue) and S (brown) atoms. These STEM images reveal that the pristine $2 \mathrm{H}$ semiconducting structure of $\mathrm{MoS}_{2}$ is preserved with minimal surface or edge defects following DGU processing ${ }^{36}$.

Atomic force microscopy (AFM) was used to characterize the thickness and size of the sorted $\mathrm{MoS}_{2}$ nanosheets. AFM images (Fig. 4a) indicate that the most buoyant fraction (f7) contains predominantly monolayers, while the less buoyant fraction (f17) primarily consists of $\mathrm{MoS}_{2}$ multilayers. From the line profile, a monolayer $\mathrm{MoS}_{2}$ flake in $\mathrm{f} 7$ has $\sim 1 \mathrm{~nm}$ thickness. This measured thickness is slightly larger than previously reported values ${ }^{2,4}$, which is reasonable due to the presence of residual F68. Figure $4 \mathrm{~b}, \mathrm{c}$ show the AFM-measured thickness and area histograms for $\mathrm{MoS}_{2}$ nanosheets from the f7, f17 and f27 fractions. These histograms were obtained from $>100$ individual
$\mathrm{MoS}_{2}$ flakes from each fraction. The average flake thicknesses for fractions f7, f17 and f27 are 1.02, 1.84 and $2.52 \mathrm{~nm}$, respectively, thus demonstrating effective thickness sorting by DGU. In contrast, the flake area histograms show significant overlap, which is consistent with the buoyant density of two-dimensional nanomaterials being independent of lateral area (to corroborate the AFM lateral size histograms, scanning electron microscopy analysis was also performed; see Supplementary Fig. 9). Overall, it is evident that the $\mathrm{MoS}_{2}$ DGU separation is driven by thickness instead of the lateral size with $\sim 86 \%$ of the $\mathrm{MoS}_{2}$ nanosheets from $\mathrm{f} 7$ having a thickness $<1.2 \mathrm{~nm}$, which corresponds to single layers of $\mathrm{MoS}_{2}$.

On the basis of the measured thickness and buoyant density values, the $\mathrm{MoS}_{2}$ experimental data are plotted with the geometrical buoyant density model in Fig. 5a. The experimental values (purple squares) imply a Pluronic F68 packing density $\sigma$ of $0.244 \mathrm{~nm}^{-2}$. Since the PPO chain of Pluronic F68 occupies an area of $\sim 1.74 \mathrm{~nm}^{2}$ on the $\mathrm{MoS}_{2}$ surface, this packing density corresponds to $\sim 42.5 \%$ surface coverage of Pluronic F68 (ref. 37). In Supplementary Table 2, the surface coverage of F68 for $\mathrm{WS}_{2}, \mathrm{MoSe}_{2}$ and $\mathrm{WSe}_{2}$ are determined to be 61.4, 68.2 and $57.0 \%$, respectively. These experimentally determined surface coverages correlate with the relative hydrophobicity of each TMD. In particular, the most hydrophilic $\mathrm{MoS}_{2}$ surface shows the lowest F68 surface coverage, which is consistent with expectations since F68 interacts with the TMD surface via its hydrophobic PPO chains.

Spectroscopic characterization. Following DGU, optical absorbance spectra for fractions f7, f17 and f27 in addition to the $\mathrm{MoS}_{2}$ a
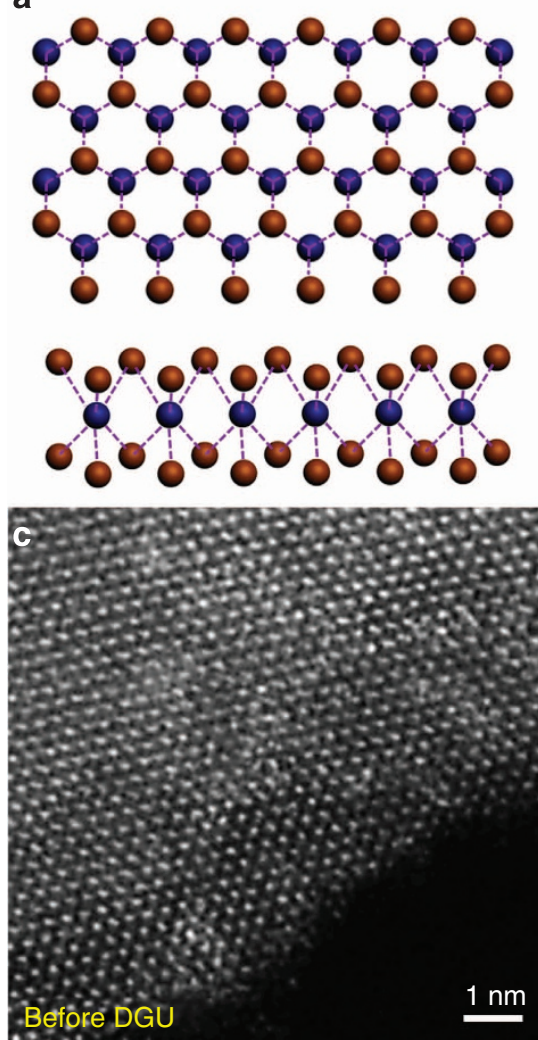
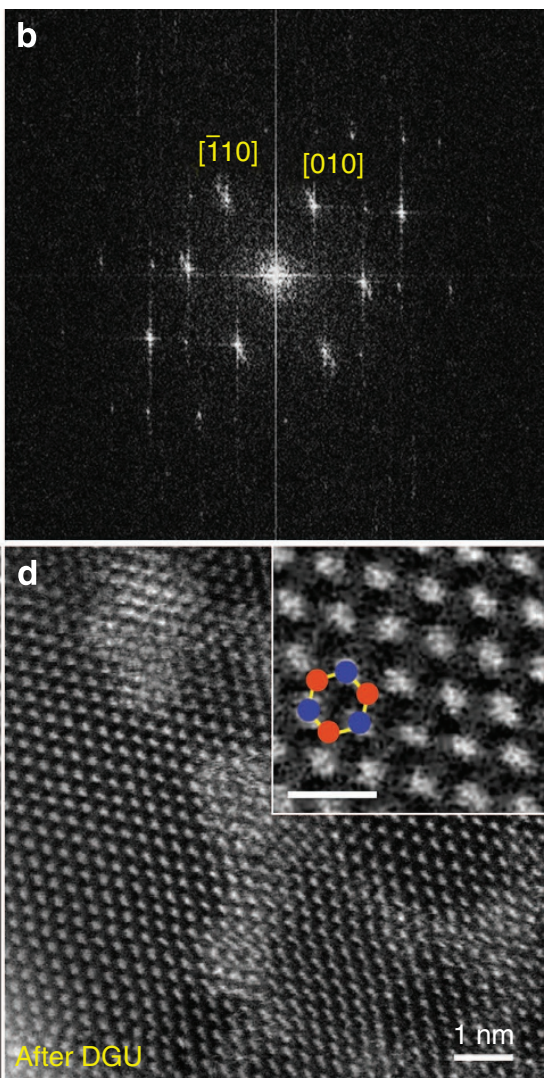

Figure 3 | Atomic structure of $\mathbf{M o S}_{\mathbf{2}}$ before and after DGU. (a) Schematic illustration of the $2 \mathrm{H}$ semiconducting crystal structure of MoS 2 shown from

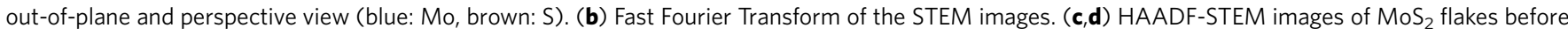
and after DGU, respectively. The inset image shows the magnified STEM image after DGU. The blue and brown spheres indicate the position of Mo and S atoms, respectively (scale bar, $0.5 \mathrm{~nm}$ ). 
a
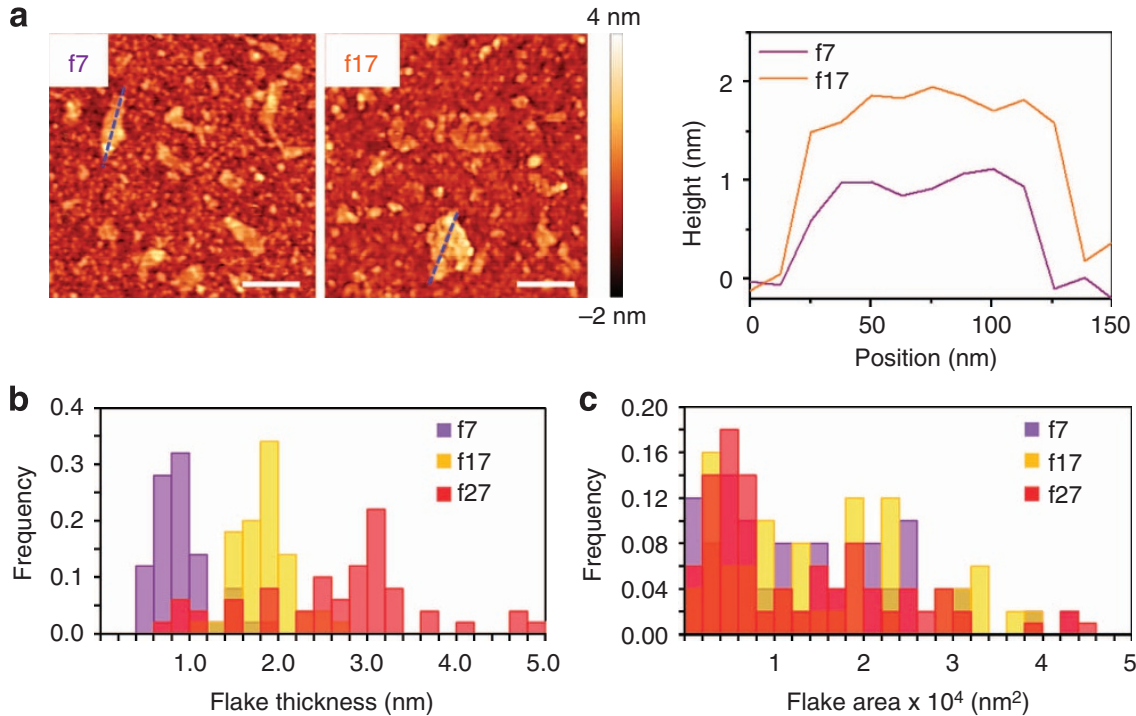

Figure 4 | Characterization of thickness and area distribution. (a) Atomic force microscopy (AFM) images of $\mathrm{MoS}_{2}$ nanosheets from f7 (purple) and f17 (orange) on a $\mathrm{SiO}_{2}$ substrate and the corresponding height profiles along the dashed lines (scale bar, $100 \mathrm{~nm}$ ). (b) Flake thickness and (c) flake area histograms for $f 7$ (purple), f17 (orange) and $f 27$ (red) fractions obtained from the AFM images.
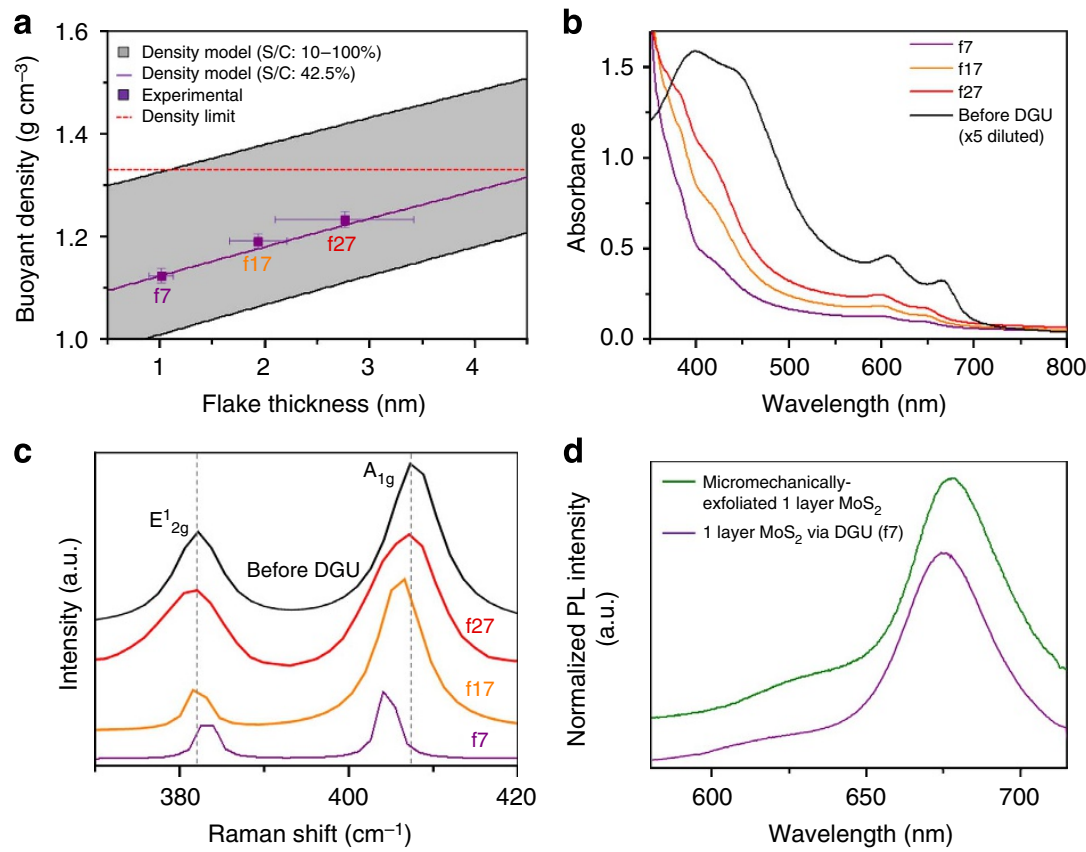

Figure 5 | Buoyant density analysis and optical properties. (a) Experimental data (purple squares) are plotted with the buoyant density model using a surface coverage variation from 10-100\% (black lines). The surface coverage of F68 on the $\mathrm{MoS}_{2}$ surface determined from the experimental data, which corresponds to $\sim 42.5 \%$, is also shown as a purple line. (b) Optical absorbance spectra of fractions f7 (purple), f17 (orange) and f27 (red), and the $\mathrm{MoS}_{2}$ dispersion before separation (black). (c) Raman spectra of each fraction showing the shifts in the in-plane and out-of-plane vibrational modes as a function of $\mathrm{MoS}_{2}$ thickness. (d) Photoluminescence spectra of fraction $\mathrm{f7}$ (purple) compared with a micromechanically exfoliated monolayer $\mathrm{MoS}_{2}$ (green).

dispersion before separation were measured (Fig. 5b). The direct $\mathrm{MoS}_{2}$ excitonic transition peaks at $\sim 610$ and $\sim 660 \mathrm{~nm}$ are present in all of the samples after DGU, further confirming that the original $2 \mathrm{H}-\mathrm{MoS}_{2}$ crystal structure was maintained through the separation process. The two absorption peaks between 600 and $700 \mathrm{~nm}$ are attributed to the excitonic peaks of $\mathrm{MoS}_{2}$. The weaker optical intensity of these peaks from f7, $\mathrm{f} 17$ and $\mathrm{f} 27$ are due to the relatively small concentration of $\mathrm{MoS}_{2}$ in the solution following DGU. In addition, the blue shift observed in these peaks is consistent with the smaller lateral flake size following DGU (See
Supplementary Fig. 9) ${ }^{38-41}$, and the strong absorption $<400 \mathrm{~nm}$ is attributed to the density gradient medium (that is, iodixanol).

Each $\mathrm{MoS}_{2}$ fraction was also characterized by Raman spectroscopy (Fig. 5c). In this case, $\mathrm{MoS}_{2}$ films were prepared by vacuum filtration and transferred onto a $\mathrm{SiO}_{2}$ substrate, at which point Raman spectra were obtained using a beam size of $\sim 1 \mu \mathrm{m}$ and an excitation wavelength of $514 \mathrm{~nm}$. The Raman spectra from the unsorted $\mathrm{MoS}_{2}$ sample and fraction f27 show two peaks, specifically the in-plane $\mathrm{E}_{2 \mathrm{~g}}$ mode at $\sim 382 \mathrm{~cm}^{-1}$ and the outof-plane $\mathrm{A}_{1 \mathrm{~g}}$ mode at $\sim 407 \mathrm{~cm}^{-1}$, which are comparable to 
those of bulk $\mathrm{MoS}_{2}$ (ref. 16). As the number of layers decreases, the $\mathrm{E}^{1}{ }_{2 \mathrm{~g}}$ mode is stiffened and the $\mathrm{A}_{1 \mathrm{~g}}$ mode is softened, such that the peaks from fraction $\mathrm{f} 7$ are shifted to $\sim 384 \mathrm{~cm}^{-1}$ and $\sim 404 \mathrm{~cm}^{-1}$, respectively ${ }^{16}$. In addition, the width of these two peaks is reduced in the more buoyant fractions, which confirms that the flake thickness homogeneity is improved by DGU.

To further verify the isolation of $\mathrm{MoS}_{2}$ monolayers, photoluminescence spectroscopy was performed on fraction $\mathrm{f} 7$ (purple line) using an excitation wavelength of $514 \mathrm{~nm}$ (Fig. 5d). For comparison, a photoluminescence spectrum was obtained under identical measurement conditions from a micromechanically exfoliated monolayer of $\mathrm{MoS}_{2}$ (green line), and both spectra were normalized using their Raman spectra (optical microscopy image of the micromechanically exfoliated monolayer $\mathrm{MoS}_{2}$ is shown in Supplementary Fig. 6). The photoluminescence spectra from each fraction show two peaks with comparable intensities at $\sim 610$ and $\sim 660 \mathrm{~nm}$ that are well correlated with the $\mathrm{MoS}_{2}$ excitonic transitions. $\mathrm{WS}_{2}$ nanosheets were similarly characterized following DGU, where photoluminescence was also observed from the most buoyant fractions (See Supplementary Fig. 7).

\section{Discussion}

In conclusion, isopycnic DGU enables solution-phase preparation of controlled thickness TMDs. Specifically, the amphiphilic block copolymer Pluronic F68 increases the hydration and thus reduces the effective buoyant density of TMDs into the range of standard density gradient media. Thickness sorting of TMDs is confirmed with AFM and Raman spectroscopy, resulting in the emergence of photoluminescence from the most buoyant single-layered nanosheets. Furthermore, atomic-resolution STEM imaging verifies that the resulting thickness-sorted TMDs possess high crystal quality with low surface and edge defect density. Since DGU has proven industrial scalability, this approach holds promise for the solution-phase production of monolayered TMDs in emerging large-volume applications including photovoltaics, catalysis, biotechnology and electronics.

\section{Methods \\ Dispersion and density gradient ultracentrifugation. One gram of $\mathrm{MoS}_{2}$ powder (American Elements) was dispersed in $70 \mathrm{ml}$ of $2 \% \mathrm{w} \mathrm{v}^{-1}$ Pluronic F68 (BASF) aqueous solution via ultrasonication using a 0.125 -inch tip in a steel beaker at $25 \mathrm{~W}$ for $2 \mathrm{~h}$. Then, $32 \mathrm{ml}$ of dispersion was carefully added on top of a $6 \mathrm{ml}$ underlayer of $60 \% \mathrm{w} \mathrm{v}^{-1}$ iodixanol and ultracentrifuged at $32 \mathrm{kr}$.p.m. for $24 \mathrm{~h}$ at $22^{\circ} \mathrm{C}$ using a SW32 Ti rotor (Beckman-Coulter). Following ultracentrifugation, $3 \mathrm{ml}$ of $60 \% \mathrm{wv}^{-1}$ iodixanol was injected to separate $\sim 10 \mathrm{ml}$ of concentrated $\mathrm{MoS}_{2}$ nanosheet solution, which was then fractionated using a piston gradient fractionator (BioComp Instruments). For the first iteration, the concentrated $\mathrm{MoS}_{2}$ solution was diluted to contain $46 \% \mathrm{w} \mathrm{v}^{-1}$ iodixanol, and placed under a linear density gradient of $25-45 \% \mathrm{w} \mathrm{v}^{-1}$ iodixanol. The linear density gradient was then ultracentrifuged at $28 \mathrm{kr}$.p.m. for $12 \mathrm{~h}$ at $22^{\circ} \mathrm{C}$. Following the first iteration, each fraction was diluted to contain $9 \% \mathrm{wv}^{-1}$ iodixanol and placed on top of a linear density gradient of $30-50 \% \mathrm{w} \mathrm{v}^{-1}$ iodixanol for the second iteration. The linear density gradient was then ultracentrifuged in an SW41 Ti rotor (Beckman-Coulter) at 41 kr.p.m. for $12 \mathrm{~h}$. All density gradient and injection solutions contained $2 \%$ $\mathrm{wv}^{-1} \mathrm{~F} 68$.}

\section{Sample preparation for scanning transmission electron microscopy. $\mathrm{MoS}_{2}$} dispersions from each step (after sonication before DGU and after DGU) were collected and placed in $20 \mathrm{k}$ MWCO dialysis cassettes (Thermo Scientific) and dialysed in $750 \mathrm{ml}$ of $2 \% \mathrm{wv}^{-1} \mathrm{~F} 68$ aqueous bath for $24 \mathrm{~h}$ to remove the density gradient medium. To remove the surfactant, $\mathrm{MoS}_{2}$ dispersions were mixed with isopropyl alcohol for $24 \mathrm{~h}$, filtered through mixed cellulose ester membranes (Millipore, $50 \mathrm{~nm}$ pore size), and rinsed with deionized water. The resulting aggregated $\mathrm{MoS}_{2}$ nanosheets were then bath sonicated (Branson ultrasonic cleaner $3510)$ to redisperse in deionized water.

Sample preparation for atomic force microscopy. The separated $\mathrm{MoS}_{2}$ fractions were dialysed in $750 \mathrm{ml}$ of $2 \% \mathrm{wv}^{-1}$ SC bath for $48 \mathrm{~h}$ to enable surfactant exchange. Before deposition, a $\mathrm{SiO}_{2}$ substrate was rinsed with acetone, methanol and deionized water and immersed in $2 \%$ polyethylenimine for $2 \mathrm{~min}$ to form a self-assembled monolayer. The substrate was then dried under nitrogen gas, rinsed in deionized water and dried again. The samples were drop casted onto polyethylenimine-treated $\mathrm{SiO}_{2}$ substrate for $5 \mathrm{~min}$, dried under nitrogen gas, rinsed in deionized water, dried again and annealed at $250^{\circ} \mathrm{C}$ for $1 \mathrm{~h}$ to remove residual surfactants and iodixanol. To increase the areal density of $\mathrm{MoS}_{2}$ flakes, this process was repeated 5-10 times.

Sample preparation for Raman and photoluminescence spectroscopy. $\mathrm{MoS}_{2}$ films were prepared via the vacuum filtration method. In particular, $2 \mathrm{ml}$ of $\mathrm{MoS}_{2}$ dispersion was filtered. The residual surfactant and iodixanol were then removed by rinsing with $30 \mathrm{ml}$ of deionized water. The resulting film was immersed in ethanol and pressed firmly onto a $\mathrm{SiO}_{2}$ substrate for $10 \mathrm{~min}$ by applying pressure with a $1.5 \mathrm{~kg}$ weight. To remove the membrane, the substrate was suspended above a boiling acetone bath at $85^{\circ} \mathrm{C}$ at a $45^{\circ}$ angle. After $15 \mathrm{~min}$, the substrate was rinsed with ethanol, acetone, ethanol again and deionized water, and then dried with nitrogen gas.

\section{References}

1. Novoselov, K. S. et al. Two-dimensional atomic crystals. Proc. Natl Acad. Sci. USA 102, 10451-10453 (2005).

2. Radisavljevic, B., Radenovic, A., Brivio, J., Giacometti, V. \& Kis, A. Single-layer $\mathrm{MoS}_{2}$ transistors. Nat. Nanotechnol. 6, 147-150 (2011).

3. Wang, Q. H., Kalantar-Zadeh, K., Kis, A., Coleman, J. N. \& Strano, M. S. Electronics and optoelectronics of two-dimensional transition metal dichalcogenides. Nat. Nanotechnol. 7, 699-712 (2012).

4. Jariwala, D. et al. Band-like transport in high mobility unencapsulated singlelayer $\mathrm{MoS}_{2}$ transistors. Appl. Phys. Lett. 102, 173107 (2013).

5. Sangwan, V. K. et al. Low-frequency electronic noise in single-layer $\mathrm{MoS}_{2}$ transistors. Nano Lett. 13, 4351-4355 (2013).

6. Kim, S. et al. High-mobility and low-power thin-film transistors based on multilayer $\mathrm{MoS}_{2}$ crystals. Nat. Commun. 3, 1011 (2012).

7. Matte, H. S. S. R. et al. $\mathrm{MoS}_{2}$ and $\mathrm{WS}_{2}$ analogues of graphene. Angew. Chem. Int. Ed. 49, 4059-4062 (2010).

8. Mak, K. F., Lee, C., Hone, J., Shan, J. \& Heinz, T. F. Atomically thin $\mathrm{MoS}_{2}$ : A new direct-gap semiconductor. Phys. Rev. Lett. 105, 136805 (2010).

9. Mak, K. F., He, K. L., Shan, J. \& Heinz, T. F. Control of valley polarization in monolayer $\mathrm{MoS}_{2}$ by optical helicity. Nat. Nanotechnol. 7, 494-498 (2012).

10. Splendiani, A. et al. Emerging photoluminescence in monolayer $\mathrm{MoS}_{2}$. Nano Lett. 10, 1271-1275 (2010).

11. Eda, G. et al. Photoluminescence from chemically exfoliated $\mathrm{MoS}_{2}$. Nano Lett. 11, 5111-5116 (2011).

12. Karunadasa, H. I. et al. A molecular $\mathrm{MoS}_{2}$ edge site mimic for catalytic hydrogen generation. Science 335, 698-702 (2012).

13. Jariwala, D. et al. Gate-tunable carbon nanotube- $\mathrm{MoS}_{2}$ heterojunction $\mathrm{p}-\mathrm{n}$ diode. Proc. Natl Acad. Sci. USA 110, 18076-18080 (2013).

14. Chhowalla, M. et al. The chemistry of two-dimensional layered transition metal dichalcogenide nanosheets. Nat. Chem. 5, 263-275 (2013).

15. Xu, M., Liang, T., Shi, M. \& Chen, H. Graphene-like two-dimensional materials. Chem. Rev. 113, 3766-3798 (2013).

16. Lee, C. et al. Anomalous lattice vibrations of single- and few-layer $\mathrm{MoS}_{2}$. ACS Nano 4, 2695-2700 (2010).

17. Sun, X. M., Luo, D. C., Liu, J. F. \& Evans, D. G. Monodisperse chemically modified graphene obtained by density gradient ultracentrifugal rate separation. ACS Nano 4, 3381-3389 (2010).

18. Nicolosi, V., Chhowalla, M., Kanatzidis, M. G., Strano, M. S. \& Coleman, J. N. Liquid exfoliation of layered materials. Science 340, 1420 (2013).

19. Coleman, J. N. et al. Two-dimensional nanosheets produced by liquid exfoliation of layered materials. Science 331, 568-571 (2011).

20. Quinn, M. D. J., Ho, N. H. \& Notley, S. M. Aqueous dispersions of exfoliated molybdenum disulfide for use in visible-light photocatalysis. ACS Appl. Mater. Interfaces 5, 12751-12756 (2013).

21. Notley, S. M. High yield production of photoluminescent tungsten disulphide nanoparticles. J. Colloid Interface Sci. 396, 160-164 (2013).

22. Zhan, Y. J., Liu, Z., Najmaei, S., Ajayan, P. M. \& Lou, J. Large-area vapor-phase growth and characterization of $\mathrm{MoS}_{2}$ atomic layers on a $\mathrm{SiO}_{2}$ substrate. Small 8, 966-971 (2012).

23. Heising, J. \& Kanatzidis, M. G. Structure of restacked $\mathrm{MoS}_{2}$ and $\mathrm{WS}_{2}$ elucidated by electron crystallography. J. Am. Chem. Soc. 121, 638-643 (1999).

24. Graham, J. M. Biological Centrifugation (Milton Park, 2011).

25. Green, A. A. \& Hersam, M. C. Processing and properties of highly enriched double-wall carbon nanotubes. Nat. Nanotechnol. 4, 64-70 (2009).

26. Arnold, M. S., Green, A. A., Hulvat, J. F., Stupp, S. I. \& Hersam, M. C. Sorting carbon nanotubes by electronic structure using density differentiation. Nat. Nanotechnol. 1, 60-65 (2006).

27. Seo, J. W. T. et al. Diameter refinement of semiconducting arc discharge singlewalled carbon nanotubes via density gradient ultracentrifugation. J. Phys. Chem. Lett. 4, 2805-2810 (2013).

28. Green, A. A. \& Hersam, M. C. Solution phase production of graphene with controlled thickness via density differentiation. Nano Lett. 9, 4031-4036 (2009). 
29. Smith, R. J. et al. Large-scale exfoliation of inorganic layered compounds in aqueous surfactant solutions. Adv. Mater. 23, 3944-3948 (2011).

30. Antaris, A. L. et al. Probing and tailoring $\mathrm{pH}$-dependent interactions between block copolymers and single-walled carbon nanotubes for density gradient sorting. J. Phys. Chem. C 116, 20103-20108 (2012).

31. Seo, J. W. T., Green, A. A., Antaris, A. L. \& Hersam, M. C. High-concentration aqueous dispersions of graphene using nonionic, biocompatible block copolymers. J. Phys. Chem. Lett. 2, 1004-1008 (2011).

32. Khan, U. et al. Solvent-exfoliated graphene at extremely high concentration. Langmuir 27, 9077-9082 (2011).

33. Paton, K. R. et al. Scalable production of large quantities of defect-free few-layer graphene by shear exfoliation in liquids. Nat. Mater. 13, 624-630 (2014).

34. Eswaraiah, V. et al. Turbulence-assisted shear exfoliation of graphene using household detergent and a kitchen blender. Nanoscale 6, 11810-11819 (2014).

35. Leon, V., Rodriguez, A. M., Prieto, P., Prato, M. \& Vazquez, E. Exfoliation of graphite with triazine derivatives under ball-milling conditions: preparation of few-layer graphene via selective noncovalent interactions. ACS Nano 8, 563-571 (2014).

36. Eda, G. et al. Coherent atomic and electronic heterostructures of single-layer $\mathrm{MoS}_{2}$. ACS Nano 6, 7311-7317 (2012).

37. Shi, H. W. et al. Surface coatings of PEO-PPO-PEO block copolymers on native and poly styrene-coated silicon wafers. Colloids Surf. A 246, 81-89 (2004).

38. Wilcoxon, J. P., Newcomer, P. P. \& Samara, G. A. Synthesis and optical properties of $\mathrm{MoS}_{2}$ and isomorphous nanoclusters in the quantum confinement regime. J. Appl. Phys. 81, 7934-7944 (1997).

39. Wilcoxon, J. P. \& Samara, G. A. Strong quantum-size effects in a layered semiconductor $-\mathrm{MoS}_{2}$ nanoclusters. Phys. Rev. B 51, 7299-7302 (1995).

40. Ou, J. Z. et al. Ion-driven photoluminescence modulation of quasi-twodimensional $\mathrm{MoS}_{2}$ nanoflakes for applications in biological systems. Nano Lett. 14, 857-863 (2014)
41. Backes, C. et al. Edge and confinement effects allow in situ measurement of size and thickness of liquid-exfoliated nanosheets. Nat. Commun. 5, 4576 (2014).

\section{Acknowledgements}

This work was supported by the National Science Foundation (DMR-1006391). This research utilized instruments in the Keck-II facility of the NUANCE center and the Keck Biophysics Facility at Northwestern University, which are supported by the NSF-MRSEC (NSF DMR-1121262) and the Keck Foundation. The STEM work was supported by the NSF Partnership for Research and Education in Materials (PREM) programme (DMR0934218), NIH RCMI Nanotechnology and Human Health Core (G12MD007591), and the Welch Foundation (Award \#AX-1615). We thank N. Mansukhani, L. Guiney, J. Zhu, K.-S. Chen and D. Jariwala for helpful discussions.

\section{Author contributions}

All the authors conceived and designed the experiments, J.K. and J.-W.T.S. performed the experiments, D.A., A.P. and M.J.Y. performed the STEM imaging and analysis, and all authors co-wrote the manuscript.

\section{Additional information}

Supplementary Information accompanies this paper at http://www.nature.com/ naturecommunications

Competing financial interests: The authors declare no competing financial interests.

Reprints and permission information is available online at http://npg.nature.com/ reprintsandpermissions/

How to cite this article: Kang, J. et al. Thickness sorting of two-dimensional transition metal dichalcogenides via copolymer-assisted density gradient ultracentrifugation. Nat Commun. 5:5478 doi: 10.1038/ncomms6478 (2014). 